\title{
Data Analytics to Enhance Game Development
}

\author{
Chananagari Prabhakar Rohit Reddy \\ Computer Science and Engineering \\ CMR Technical Campus \\ Hyderabad, Telangana, India
}

\author{
Buchi Avinash \\ Computer Science and Engineering \\ CMR Technical Campus \\ Hyderabad, Telangana, India
}

\begin{abstract}
Recently, the video game industry revenue increased significantly, with this intensely growing sector breeds more complex and sweeping gaming formats, including desktop, mobile, console, VR, and others. For what it's worth, data analysts and business intelligence developers are turning out to be really useful staff in gaming companies. These various format bring a surge of multi-source user data with them: play time, user's interactions, quitting points, and gaming style are just a portion of the metrics there to gather. Data analysts and BI developers can examine and change these and others into important insights. Thus, with these insights on hand, gaming companies can get the hang of situating their product better, planning increasingly vivid games, bringing more personalization, and, similarly as significant, producing more profits.
\end{abstract}

Keywords-Data analytics, Key performance indicators, enhancing game design, data driven, monetization.

\section{INTRODUCTION}

Data analytics is relevant all through the cycle of your game and is utilized by both outside the indie engineers and enormous studios. At release, you can utilize data analytics to see how you're procuring users and where to focus. During game development, you can figure out what keeps players connected with, what different groups of players like and aversion about your game and tune it to match their inclinations. This game cycle is pertinent regardless of what size you are or whether you're a publisher or studio. Some of the most recent games like Fortnite, Mortal Kombat and now Mario Kart are the games which are utilizing data and analytics to improve game development and attract more players on to the platform. Because of the exponential growth in mobile gaming, games are creating huge amount of data that can be tapped for collecting insights. Let's consider most popular ways of how today's gaming companies can augment their capabilities with data analytics, where analytics alongside data science is deployed for game development

\section{TECHNIQUES FOR OBJECTIVE IMPACT}

The Most games usually expect players to follow a specific strategy; they have an 'objective' which should be finished to go to the following level in the game. Good strategies and gaming help accomplish this. However, the gaming performance relies upon the players. For example, in specific games particularly of the open-world genre, in-game movements and actions are completely done by players and lesser affects the primary objective. These cannot be changed or modified. In basic words, how you navigate in games like
Grand Theft Auto, Assassin's Creed or Far Cry is your decision given you don't diverge from the game objectives. Just the strategy part can be devised from data science techniques. For instance, Civis Analytics features how one can pick the best Mario Kart character to win a race from techniques, such as Pareto optimality and exploratory data analysis. It also charts out different configurations like blends of game objects, such as characters, karts, and tire that can be made in every possible ways. Based on this, clearly you pick the best configuration but once you start a race in the game, winning is totally subject to your style of playing.

\section{ENHANCING GAME DESIGN}

Data analytics additionally enables gaming companies to help game design. Building interactive and complex situations for games requires a huge stock of innovativeness, however it also needs a legitimate understanding of what functions admirably for the audience. Here's the data analytics can lend some assistance. For example, analytics assists companies with recognizing problematic ongoing game play moments for users. To be sure, data can show that a few levels may be excessively dull, some may be excessively challenging, and some may essentially contain bugs that don't let users push ahead.

As an ever increasing number of data is created on the web, data analytics can help set trends and guarantee players return to platform. Coincidentally, this is what happened to King Digital Entertainment. This well known game designer once caught an unanticipated issue with its most popular game, Candy Crush Saga. Users were hugely abandoning level 65, reasons obscure. With 725 levels altogether, for Candy Crush Saga such a propensity was a serious trouble. King went to data analysts to uncover that most people were abandoning a specific gaming element that didn't let users make it past level 65 , they showed that this game is classified as a 'NP-hard problem' evident in computational complexity theory when users are in level 65. After certain enchantment in the development division, the component was erased, and user retention got going again.

Here's another instance, Valve Software, a gaming organization behind such hits as Half-Life and DOTA, is an innovation pioneer in the gaming scene. For instance, it utilizes deep learning to avoid fraud in its games and identify cheaters. With respect to data analytics, the company utilized its capacity in no less striking manner. Valve's other top game, is a challenge between two teams of five. The company gathered and analyzed certain user data, including which firearms the teams picked, how their behavior changed during the game, how they killed, and how they died. They did it to calibrate the 
game balance and to ensure that no specific team is overpowered in view of staying focuses in game design. Therefore, they created a more pleasant game with a great balance between teams. All things considered, as data analytics enables gaming companies to work out the crimps of game design, users get gaming experience that keep up with their skills.

\section{TRACKING KEY PERFORMANCE INDICATORS}

In their endeavor to precisely measure a game's overall performance, makers definitely face the need to respond to some fairly pressing inquiries. What is the number of day by day dynamic users in a game? What numbers of dynamic players are there month to month? Were there any new users a month ago? On the off chance that indeed, what number of? These inquiries are lined up with the most fundamental KPIs of gaming analytics, including daily active users, monthly active users and average revenue per user. Figuring them with data analytics and visualizing with Business Intelligence tools can help answer the inquiries listed above.

Besides, companies can more likely comprehend the explanations behind a game's high points and low points, and construct increasingly powerful strategies. The magnificence of utilizing data analytics toward understanding these KPIs is that it additionally permits tracking certain trends, positive and negative the same. For instance, if a game attracts new users every day, the likelihood that some of them will upgrade to a paid account ascents exponentially. Realizing that, gaming companies may some way or another reexamine their pricing policy. Also, degrading monthly active users rates may discuss approaching users wearing down, which is as yet conceivable to fight off whenever recognized in time.

\section{INCREASING MONETIZATION}

Figure Data analytics likewise enables gaming companies to perceive what precisely brings them more money and, consequently, modify their monetization methodologies accordingly. To be sure, if a company uncovers that numerous users lean toward altering their armor or weapon, it's really sensible to offer them in-game armor and weapon upgrade.

However, it's not just about weapons and armor, and Zynga's example is here to demonstrate it. This gaming company's predominant plan of action was allowed to play freely, however it likewise offered a premium, ad-free account. The issue was that typically just $2 \%$ of players really paid. Fortunately, the company discovered its approach to soar paid memberships, all with the assistance of data analytics. In reality, in the first version of Farmville, one of their most well known games, users loved interacting with animals that were at first just decorations. A few users even began acquiring animals with real money, so in the accompanying version of the game, Zynga made animals a focal feature and even made 'uncommon species' to animate users additionally.
Such a data driven methodology toward monetization not just shows high return of investments (ROI) for gaming companies; it likewise hits chord with users. The latter are offered precisely what they need from a game, and that is the way game makers can deliver customized, better-focused on features and products.

\section{DATA-CONTROLLED GAME DEVELOPMENT}

Game design additionally plays a significant job in keeping players snared to the platform. Having magnificent graphics and a superior game play won't get the job done. Insights from gaming data are exceptionally considerable. Adam Fletcher of Gyroscope Software perfectly puts it thusly, "It can appear to be odd to think about games, frequently described as aesthetic endeavors, as something you can quantify and tune progressively like a machine. Data science is no substitution to innovativeness and design. It is a supplement to game design that can bolster those endeavors and fill in holes that design cannot."An explicit area in Data Science, for instance, data warehousing or data engineering, probably won't be required for offline games. Moreover, Data science ought to likewise answer game related situations since each game is unique. Another zone which gaming companies explore is extending the install base. Likewise, in certain examples, game may have a huge user base however may not have many sessions or highlight low session length when contrasted with the prelaunch estimates. This implies that players are no longer keen on the game. Data science techniques, particularly personalization can be utilized to increase user base and push up budgetary KPIs.

\section{CONCLUSION}

Actually, to make data analytics work without limit, gaming companies ought to eagerly make a beeline for setting up a data driven culture. Gathering, binding together, visualizing, cleaning, and afterward dissecting data is an entire large undertaking task on its own. The individuals who are as of now on their way toward data based decision making, however, should remain patient and determined. At some point or another, they'll see: actualizing data analytics is the effort that pays off, and the gaming industry has numerous examples to bear this out.

\section{REFERENCES}

[1] https://www.ics.uci.edu/ eppstein/cgt/hard.html

[2] https://towardsdatascience.com/why-business-product-should-alwaysdefine-kpis-goals-for-data-science-450404392990

[3] https://medium.com/civis-analytics/the-best-mario-kart-characteraccording-to-data-science-7dfb65d4c18e

[4] https://arxiv.org/pdf/1403.1911.pdf

[5] https://www.datanami.com/2018/07/31/inside-fortnites-massive-dataanalytics-pipeline/

[6] https://www.iflexion.com/blog/ai-in-games

[7] https://www.iflexion.com/business-intelligence 